\title{
Research on Magnetic Bead Motion Characteristics based on Magnetic Beads Preset Technology
}

\author{
Zhao Li ( $\nabla$ lzwhale@163.com ) \\ Henan University of Science and Technology \\ Xiangyang Zu \\ Henan University of Science and Technology \\ Zhe Du \\ Henan University of Science and Technology \\ Zhigang Hu \\ Henan University of Science and Technology
}

\section{Research Article}

Keywords: microfluidic control, magnetic beads preset, permanent magnet, magnetic bead control, motion characteristics

Posted Date: July 1st, 2021

DOI: https://doi.org/10.21203/rs.3.rs-668578/v1

License: (c) (i) This work is licensed under a Creative Commons Attribution 4.0 International License. Read Full License 


\title{
Research on Magnetic Bead Motion Characteristics based on Magnetic Beads Preset Technology
}

\author{
Zhao $\mathrm{Li}^{1 *}$, Xiangyang $\mathrm{Zu}^{2}$, Zhe $\mathrm{Du}^{2}$, Zhigang $\mathrm{Hu}^{2}$
}

1. Department of Packaging Engineering, Henan University of Science and Technology, Luoyang, Henan, China

2. School of Medical Technology and Engineering, Henan University of Science and Technology, Luoyang, Henan, China

* Corresponding author

E-mail:1zwhale@163.com

\begin{abstract}
In order to improve the detection efficiency and accuracy of microfluidic chip, a magnetic beads preset technology were designed by using double permanent magnets as external magnetic field and the motion characteristics of preset magnetic beads were studied. The control principle of magnetic beads preset technology was introduced in detail, and the control structure was designed. The coupled field characteristics for magnetic beads in microchannels were analyzed, and the motion models of magnetic beads were established based on the bead preset technology, including capture motion and mixing motion. The relationship between the magnetic field force and the flow velocity for capturing magnetic bead, and the mixing time under the influence of flow field and magnetic field were derived. The magnetic beads capture effect of magnetic beads preset technology was verified by experiments. The study showed the magnetic beads preset technology can better realize the accuracy and efficiency of the magnetic bead control in the microchannel. The derivation of the magnetic bead motion model can understand the motion characteristics of the magnetic bead more clearly, facilitate accurate control of the magnetic bead, and improve the success rate of the microfluidic detection.
\end{abstract}

Key words: microfluidic control; magnetic beads preset; permanent magnet; magnetic bead control; motion characteristics

\section{Introduction}

Magnetic bead is one kind of magnetic material with particle size between 1 100nm, which not only has unique surface effect, volume effect, quantum size effect, functional groups like other general nano material, but also exhibits superparamagnetism, magnetic responsiveness, high coercivity ${ }^{1}$, and can be controlled by external magnetic field. At present, magnetic bead has been attracted more and more attention ${ }^{2-5}$, and are widely used in biomedical science ${ }^{6-11}$. For example, 
the magnetic beads with special surface treatment can be used to form a new complex by nonspecific or specific combination with the corresponding target materials, and be separated under the control magnetic field, than the separated magnetic beads are eluted by the eluent to complete DNA extraction ${ }^{12-13}$. The key of magnetic bead control is to set a reasonable magnetic field to achieve magnetic beads effectively captured and fully mixed with the reaction solution under the flow field influence. This paper presents a magnetic beads preset technology, analyzing the feasibility of magnetic beads preset technology and the motion characteristics of preset magnetic beads, in order to improve the efficiency of microfluidic detection and simplify the microfluidic detection equipment.

\section{Analysis of magnetic bead control}

\subsection{Magnetic bead control motion}

The functions of magnetic beads are different in microfluidic detection, but they all need to be controlled precisely in micro scale and enhance mass transfer efficiency by use of magnetic field. Therefore, the dynamic characteristics and transmission pattern of magnetic beads in the micro channel under the effect of magnetic field are the important research on the control of magnetic beads ${ }^{14-16}$. The control motions about magnetic beads capture and magnetic beads mixing with the solution were focused on our study, and the control motion models are shown in Fig. 1, which the capture efficiency and mixing efficiency are the most important research problems for the two kinds of motion.

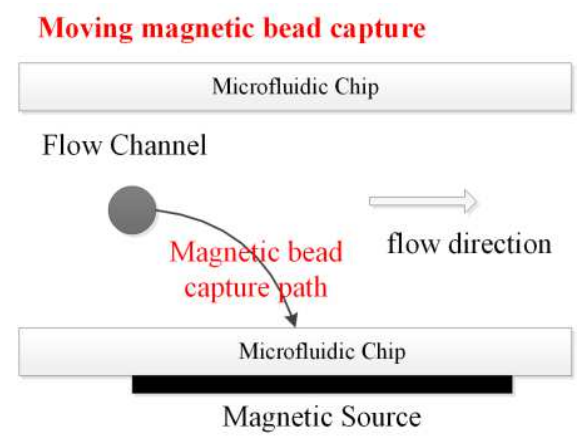

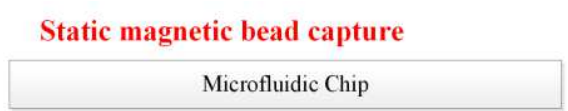

Flow Channel

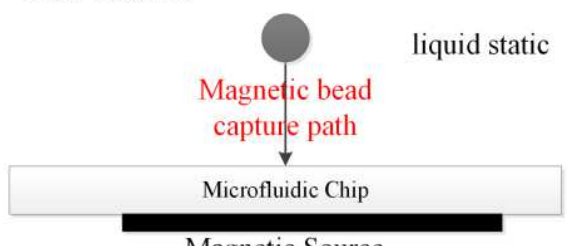

Magnetic Source

a. Magnetic bead capture

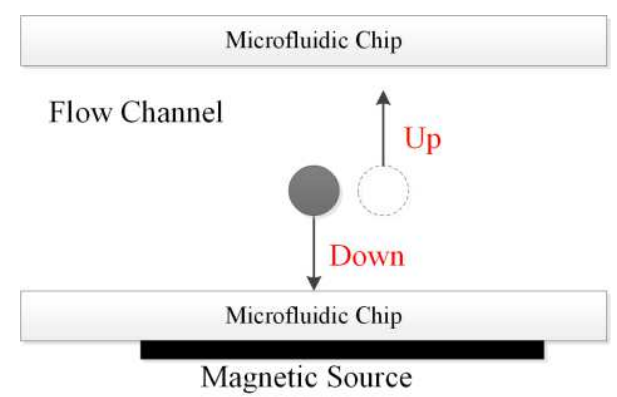

b. Magnetic bead mixing with the solution

Fig. 1 Magnetic bead control motion 


\section{(1) Capture efficiency}

Magnetic beads are generally exogenous for microfluidic chips, so magnetic beads need to be captured to the designated position in chip channel and wait for the subsequent reactions, as shown in Fig. 1a. The magnetic beads capture efficiency mainly includes the number of magnetic beads captured and the time needed to complete the capture. The effective number of magnetic beads captured can ensure the success rate of detection, and the reasonable capture time can improve the detection efficiency. Magnetic beads capture efficiency is an important parameter in micro scale biological detection, which is mainly affected by gradient of magnetic force ${ }^{17}$ and fluid viscous resistance ${ }^{18}$. In our study, the magnetic beads were set to be endogenous for microfluidic chip, which can fully ensure magnetic beads capture efficiency.

\section{(2) Mixing efficiency}

The magnetic beads mixing motion refers to the relative motion between magnetic beads and solution, as shown in in Fig. 1b. Because the Reynolds number of fluid in microchannel is very small (generally less than 10), so the conventional turbulent mixing cannot be occurred. If the fluid mixing only depends on diffusion without external disturbance, the mixing time will be too long and the mixing effect will be insufficient. Too long mixing time will lead to low efficiency of the detection system, and insufficient mixing will affect the accuracy of the detection system. According to the existence of external power or not, the mixing process of magnetic beads and solution can be divided into passive mixing and active mixing. Compared with passive mixing, the mixing effect of active mixing is more ideal and easy to control, so the active mixing was used as the control method of magnetic beads mixing motion in our study.

\subsection{Selection of control magnetic field}

According to the differences of magnetic field sources, the research about magnetic bead control technology is mainly divided into two categories: micro-electro-mechanical system(MEMS) and external permanent magnet system. Generally speaking, although the MEMS technology has higher integration, but exists the following problems ${ }^{19-20}$ : (1) The integrated coil will generate heat, and a new heat source will be introduced on the chip, which will be difficult to adapt to the temperature sensitive sample; (2) The magnetic induction generated by MEMS is not high, so the separation efficiency is low; (3) The processing of MEMS is complicated and difficult; (4) The chip used MEMS is opaque usually, which will be unfavourable for optical detection. However, the external permanent magnet system is relatively simple, which do not affect microchannel design and is easy to achieve magnetic field control. In our study, a kind of external permanent magnet system was designed and the motion characteristics of magnetic beads based on the system were analyzed. 


\section{Magnetic beads preset technology}

Magnetic beads preset technology is that the magnetic beads were put into the microfluidic chip in advance. When the reaction occurred, magnetic beads could directly participate in the reaction. Magnetic beads preset technology mainly includes the method of magnetic beads preset and the control structure of preset magnetic beads.

\subsection{Magnetic beads preset microfluidic detection process}

Microfluidics is a technology using microchannels with sizes of 10-100 $\mu \mathrm{m}$ to handle or manipulate microfluidics ${ }^{21-22}$. Microfluidics detection is a micro total analysis system achieving a series of biochemical experiments on a microchip, such as biological samples preparation, DNA extraction, PCR reaction, on-chip detection and so on ${ }^{23-25}$. Compared with traditional detection methods, microfluidic detection has obvious advantages. Microfluidic can handle a small number of samples to speed up the synthesis and analysis, reduce the consumption of reagents; The high surface area mass ratio and heat transfer coefficient of microfluidic can significantly reduce the time required for reaction and analysis to improve detection efficiency; The laminar flow characteristics in microfluidics can accurately control and optimize the fluid motion; Microfluidics devices are compact, miniaturized, highly automated, and allow parallel analysis, which can achieve high-throughput, large-scale and rapid detection.

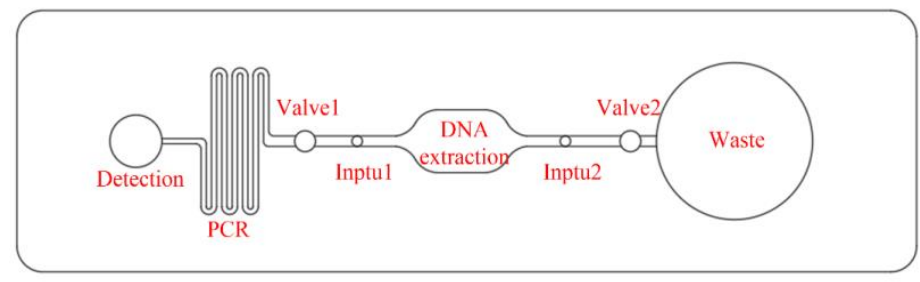

Fig. 2 Structure of microfluidic chip with preset magnetic beads

The platform of microfluidic detection is microfluidic chip ${ }^{26-28}$. The structure of microfluidic chip designed with preset magnetic beads is shown in Fig. 2. The detection process is as follows: (1) Close valve 1 and open valve 2 , the distilled water was injected from inlet 1 , then the preset magnetic beads in the DNA extraction area began to dissolve and release. After magnetic beads completely releasing and capturing, the sterile air was introduced into microchannel from inlet 1 for purging; (2) Close valve 2 and open valve 1, sample was injected from inlet 2 and fully mixed with magnetic beads by the control of magnetic field; (3) Close valve 1 and open valve 2, sterile air was introduced into microchannel again from inlet 1 for purging; (4) Close valve 2 and open valve 1, eluent was injected from inlet 2 and fully mixed with magnetic beads by the control of magnetic field; (5) Close valve 2 and open valve 1, the pushing liquid was injected from inlet 2 to push the eluent to the PCR reaction area; (6) After the eluent completing the PCR reaction, the eluent was pushed to the detection pool by pushing liquid for fluorescence detection; (7) Display the detection results, and complete the microfluidic detection. 


\subsection{Magnetic beads preset method}

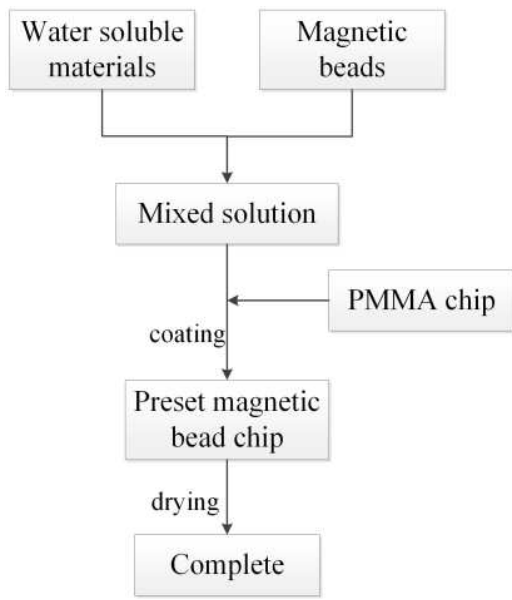

Fig. 3 Magnetic beads preset process

The process of magnetic beads preset is shown in Fig. 3, and the detailed implementation steps are as follows:

(1) Preset coating mixing. Putting coating solution (such as PVA) and magnetic beads into vortex oscillator to mix fully and to ensure mixing solution uniform density.

(2) Preset coating spraying. The quantitative mixing solution was evenly coated on the bottom of magnetic beads preset groove on the chip, and the thickness of the magnetic beads preset coating can be adjusted as needed, but it should not exceed $1 / 3$ of the magnetic bead preset groove depth.

(3) Preset coating drying. Placing the chip with preset coating into the blast drying oven at $50{ }^{\circ} \mathrm{C}-70{ }^{\circ} \mathrm{C}$ for $20 \mathrm{~min}-30 \mathrm{~min}$. After the water in PVA solution was volatilized, the magnetic beads preset coating will be formed, which magnetic beads were wrapped by magnetic beads preset coating and effectively adhered to the bottom of magnetic bead preset groove.

(4) Microfluidic chip assembly. Making the film bonding on the chip to complete preset magnetic beads encapsulation, and pasting the permanent magnet on the back of the chip to complete the assembly of magnetic beads preset microfluidic chip.

\subsection{Preset magnetic beads control structure}

The functions of preset magnetic beads control structure mainly include: preset magnetic beads capture during the dissolution of magnetic beads coating; the full mixing between preset magnetic beads and reaction solution.

\section{(1) Preset magnetic beads capture}




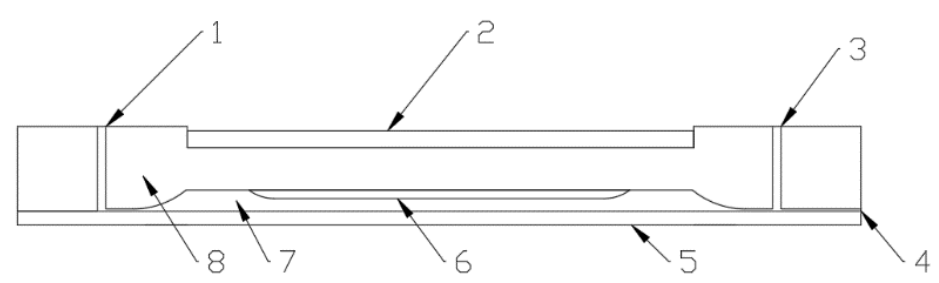

1. Liquid inlet 2. Preset permanent magnet 3. Waste liquid outlet 4. Eluent outlet

5. Film 6. Magnetic beads preset coating 7. Magnetic beads preset groove 8. Chip

Fig. 4 Magnetic beads preset microfluidic chip

Fig. 4 shows the structure of microfluidic chip with preset magnetic beads. When the detection began, distilled water was injected into and filled the magnetic beads preset groove 7 from liquid inlet 1 with eluent outlet 4 closed by valve (not shown in the figure) and the waste liquid outlet 3 opened. So the magnetic beads preset coating 6 would be placed in distilled water and after a while, the water-soluble material in the magnetic beads preset coating was dissolved, then the magnetic beads wrapped were released. Due to the magnetic force of preset permanent magnet 2 , the magnetic beads were captured at the bottom of magnetic beads preset groove.

\section{(2) Preset magnetic beads mixing}

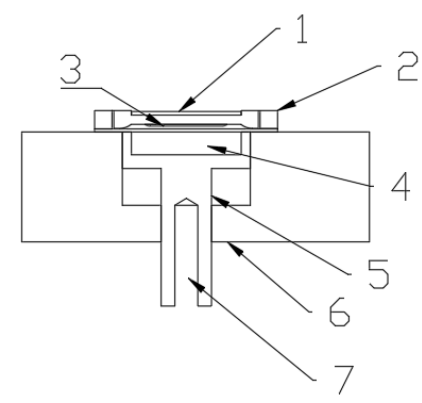

1. Preset permanent magnet 2. Chip 3. Film 4. Movable permanent magnet

5. Movable permanent magnet bracket 6. Base 7. Screw deceleration stepper motor hole

Fig. 5 Magnetic beads mixing motion control structure

Fully mixing between magnet beads and adsorbent (sample solution) or eluent is the premise to ensure the effect of adsorption or elution. Magnetic beads preset technology used double-sided permanent magnet system, and the magnetic beads mixing motion was realized by the difference of magnetic field intensity caused by the interaction between preset permanent magnet (fixed weaker permanent magnet) and the movable permanent magnet (stronger permanent magnet). As shown in Fig. 5, magnetic beads preset microfluidic chip was fixed on the base 6 with a motion guide groove for the movable permanent magnet bracket 5 to ensure the up and down motion of movable permanent magnet bracket. The up and down motion of movable permanent magnet 4 was achieved by screw deceleration stepping motor (not shown in the figure), which matched with the thread hole at the lower end of movable permanent magnet bracket 5 .

After the magnetic beads preset coating dissolved, the magnetic beads were forced on the bottom of magnetic beads preset groove by preset permanent magnet 1 , and the initial position of movable permanent magnet 4 was located at the bottom of base guide groove, so the influence of movable permanent magnet on the magnetic beads could be ignored. After the adsorbent or eluent was 
filled, the movable permanent magnet bracket 5 and the movable permanent magnet 4 were raised. Because the magnetic field strength of movable permanent magnet was stronger greatly than that of the preset permanent magnet, the adsorption force of movable permanent magnet for the magnetic beads gradually increased with the rise of movable permanent magnet, and the magnetic beads began to leave the bottom of the magnetic beads preset groove and moved towards the film direction, that will realize the downward mixing of the magnetic beads with the adsorbent or eluent. When the movable permanent magnet moved downward, the adsorption force for magnetic beads gradually weakened, and the adsorption force of preset permanent magnet for magnetic beads became the main adsorption force again, so the magnetic beads moved to the bottom of magnetic beads preset groove, that will realize the upward mixing between the magnetic beads and the adsorbent or eluent. The control of magnetic beads mixing motion could be adjusted by changing the motion speed for adjusting mixing speed, by changing the size of movable permanent magnet for adjusting the motion track of magnetic beads and by changing the relative position of movable permanent magnet and preset permanent magnet to achieve the suspension distribution of magnetic beads.

\subsection{Advantages of magnetic beads preset technology}

\section{(1) Achieving the accurate control of magnetic beads using amount}

The coupling effect between flow field and magnetic field should be considered for magnetic beads capture in microfluidic chip, so the motion of magnetic beads capture is complex, and it is difficult to accurately control the amount of magnetic beads capture ${ }^{29}$. When the capture amount is insufficient, the detection requirements are hard to satisfy. When the capture amount is too large, the detection cost will increase, which will affect the promotion of microfluidic detection technology. The magnetic beads preset technology can accurately quantify the magnetic beads in the chip to effectively ensure the optimal amount of magnetic beads using for microfluidic detection.

\section{(2) Simplify microfluidic devices}

Microfluidic detection involves different reaction liquid supply, different mechanical control devices design (such as pump, valve), and different field control systems design (such as optical, thermal, magnetic field system). However, the size of microfluidic chip is very small, which leads to the higher integration of microfluidic detection device, so the operation structure design for microfluidic chip is more complex due to the limitation of space. And if a certain operation structure for microfluidic chip could be reduced, it will greatly simplify the microfluidic devices. Using magnetic beads preset technology, the magnetic beads are pre-placed in the chip, reducing the magnetic beads feedings mechanism and the complexity of microfluidic device. 


\section{Analysis of magnetic beads preset technology effect}

\subsection{Magnetic beads preset coating}
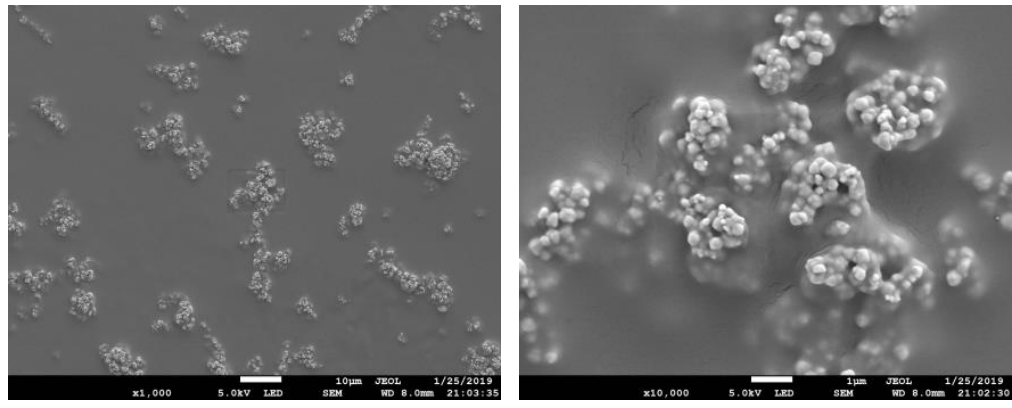

Fig. 6 Scanning electron microscope of magnetic beads preset coating

The scanning electron microscope (SEM) of magnetic beads preset coating is shown in Fig. 6. It can be seen that the magnetic beads were tightly wrapped by water-soluble materials and attached to the bottom of magnetic beads preset coating groove. In this experiment, polyvinyl alcohol (PVA) was selected as water-soluble material, which has good biocompatibility and without environmental pollution.

\subsection{Preset magnetic beads dissolution and capture}

For the convenience of observation, quartz glass square hole capillary was used as the carrier for preset magnetic beads to observe the effect of dissolution and capture.

(1) Materials and equipment: Quartz glass square hole capillary with inner diameter of $1 \times 1 \mathrm{~mm}$; Nd-Fe-B strong permanent magnet with external dimension of $20 \times 10 \times 3 \mathrm{~mm}$; Video microscope, ZX-H1400, Shenzhen Zhongwei Kechuang Technology Co., Ltd.

(2) Experimental methods: A wire with smooth surface, good centrality and diameter of $0.6 \mathrm{~mm}$ was placed in the quartz glass tube in advance. A certain concentration of PVA solution mixed evenly with magnetic beads was inhaled into a needle tube, then the PVA/magnetic beads mixture were injected into the quartz glass tube. After drying, the wire was carefully taken out from the quartz glass tube to form a preset magnetic beads quartz glass tube. The quartz glass tube was placed in the self-made bracket with one end connected with the plunger pump and the other end led into the beaker through the teflon tube. A permanent magnet was set under the quartz glass tube, and a video microscope was set in front of the quartz glass tube. When all the preparatory work is done, start the plunger pump to make the distilled water pass through the quartz glass tube at a certain flow rate to observe the dissolution effect. 

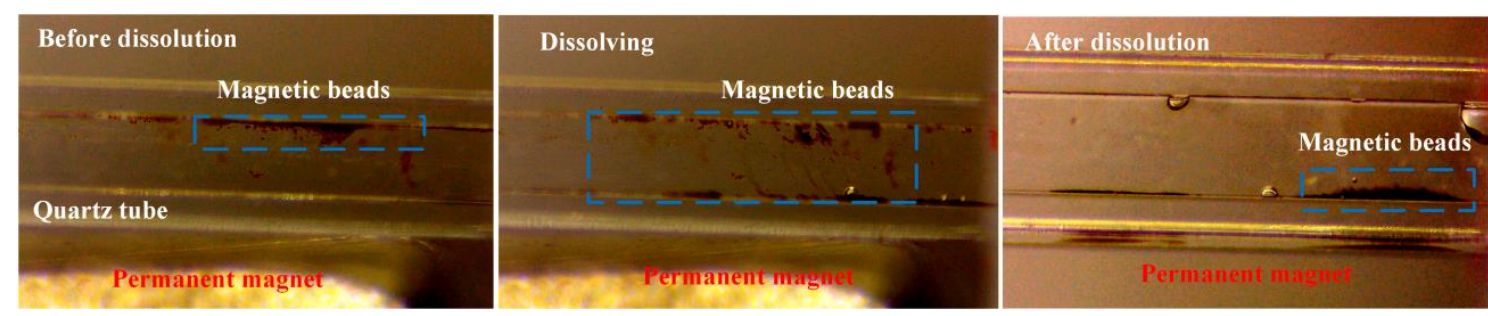

Fig. 7 Dissolution effect

The dissolving effect of preset magnetic beads is shown in Fig. 7. The dissolution rate of magnetic beads is related to the amount of preset magnetic beads, the solubility of PVA solution, the size of permanent magnet and the flow rate. The experimental results showed that the preset magnetic beads can be completely dissolved and captured within 20 s by setting reasonable parameters.

\subsection{Function verification of preset magnetic beads}

Whether the function of magnetic beads mixed with PVA solution changed should be verified. Through the relevant experiments, it can be confirmed that the detection effect of magnetic beads dissolved from the PVA solution were consistent with those of normal magnetic beads, indicating that the magnetic beads dissolved from the PVA solution do not affect their surface function.

\section{Analysis of preset magnetic beads motion characteristics}

\subsection{Coupled field analysis}

The dynamic characteristics of magnetic particles are mainly determined by two factors in microfluidic chips, including the gradient magnetic field applied externally and the flow field in the microchannel. Therefore, the motion characteristics of magnetic beads in the microchannel mainly involves the theory of magnetostatics, hydrodynamics and Newton's second law.

\section{(1) Magnetic field}

Magnetic field is the main way to control material transport in microfluidic chip. Compared with electric field, magnetic field has unique advantages: Magnetic force is not affected by sample concentration, $\mathrm{pH}$ value and other parameters; No heat is generated by permanent magnet; Magnetic field can penetrate most of the materials such as glass and polymer ${ }^{30}$; Magnetic field is mild and non-destructive to most living cells. In magnetic beads preset technology, compound magnetic field effect of different permanent magnets on the magnetic beads is mainly considered.

\section{(2) Flow field}

When the magnetic beads enter the chip from external, the capture efficiency of magnetic beads is closely related to the flow rate. When the flow rate is low, the capture efficiency is high, but the 
efficiency of biological analysis system is low. When the flow rate is high, the capture efficiency is low. The magnetic beads preset technology can weaken the influence of flow rate, focus on the influence of static flow field on magnetic beads, and simplify the analysis model.

\subsection{Mathematical model of magnetic beads motion}

The external forces on magnetic beads in the microchannel include magnetic force, fluid viscous force, interaction force between particles, gravity and buoyancy ${ }^{31-32}$. According to Newton's second law:

$$
m \frac{d^{2} \tau}{d t^{2}}=F_{m}+F_{d}+F_{g}+F_{f}
$$

Where $m$ is the mass of particle, $\tau$ is the position vector diameter of particle, and $F_{m} 、 F_{d} 、 F_{g} 、 F_{f}$ are the magnetic force, viscous force, gravity and buoyancy. Because magnetic force and viscous force are much greater than gravity and buoyancy, the model is simplified as formula (2), ignoring gravity and buoyancy.

$$
m \frac{d^{2} \tau}{d t^{2}}=F_{m}+F_{d}
$$

The forces on magnetic bead are shown in Fig. 8. The magnetic field force is decomposed into three components $\left(F_{x}, F_{y}, F_{z}\right)$.

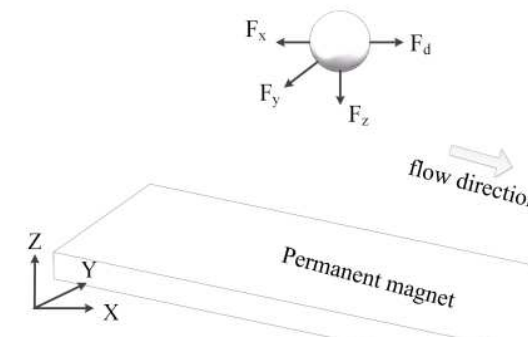

Fig. 8 Forces of magnetic bead

\section{(1) Magnetic force $F_{m}$}

Magnetic force $F_{m}$ can be expressed as:

$$
F_{m}=\mu_{0} M V_{p} \nabla H
$$

Where $\mu_{0}$ is the vacuum permeability, $M$ is the field dependent magnetization of magnetic bead, $V_{p}$ is the volume of magnetic bead, and $H$ is the space magnetic field strength. When the particle is saturated magnetized, its field dependent magnetization is the saturation magnetization, that is, $M$ $=M_{s}$.

In general, the field dependent magnetization follows the formula:

$$
M=f(H) H
$$

Where $H$ is the mode of magnetic field intensity, $H=|h|$, and $f(H)$ satisfies the equation: 


$$
f(H)=\left\{\begin{array}{l}
\frac{3 \chi}{3+\chi}, H<\frac{\chi+3}{3 \chi} M \\
\frac{M_{s}}{H}=\chi, \quad H \geq \frac{\chi+3}{3 \chi} M
\end{array}\right.
$$

Where, $V_{p}=\frac{3}{4} \pi r^{3}, \chi$ is the magnetic susceptibility of the magnetic bead, and $R$ is the radius of the magnetic bead.

Because $H=\frac{B}{\mu_{0}}$, so:

$$
F_{m}=\frac{1}{\mu_{0}} f(H) V_{p} B \nabla B=\frac{1}{\mu_{0}} f(H) V_{p}\left(B_{x} \frac{\partial B}{\partial x}+B_{y} \frac{\partial B}{\partial y}+B_{z} \frac{\partial B}{\partial z}\right)
$$

Where $B$ is the magnetic flux density.

The magnetic field force on magnetic bead depends not only on the magnetic induction intensity, but also on the magnetic field gradient. Magnetic bead tends to move towards the maximum magnetic field intensity in the non-uniform magnetic field. The magnetic force in different directions are as follows:

$$
\left\{\begin{array}{l}
F_{\mathrm{x}}=\frac{1}{\mu_{0}} f(H) V_{p} B \nabla B=\frac{1}{\mu_{0}} f(H) V_{p}\left(B_{x} \frac{\partial B_{x}}{\partial x}+B_{y} \frac{\partial B_{x}}{\partial y}+B_{z} \frac{\partial B_{x}}{\partial z}\right) \\
F_{y}=\frac{1}{\mu_{0}} f(H) V_{p} B \nabla B=\frac{1}{\mu_{0}} f(H) V_{p}\left(B_{x} \frac{\partial B_{y}}{\partial x}+B_{y} \frac{\partial B_{y}}{\partial y}+B_{z} \frac{\partial B_{y}}{\partial z}\right) \\
F_{z}=\frac{1}{\mu_{0}} f(H) V_{p} B \nabla B=\frac{1}{\mu_{0}} f(H) V_{p}\left(B_{x} \frac{\partial B_{z}}{\partial x}+B_{y} \frac{\partial B_{z}}{\partial y}+B_{z} \frac{\partial B_{z}}{\partial z}\right)
\end{array}\right.
$$

\section{(2) Viscous force $F_{d}$}

In microfluidic system with low Reynolds number, the viscous force acting on particles can be obtained by Stokes law:

$$
F_{d}=6 \pi \eta r\left(v_{f}-v_{p}\right) f_{D}
$$

Where, $\eta$ is the dynamic viscosity of fluid, $r$ is the radius of magnetic bead, $v_{f}$ is the velocity of fluid, $v_{p}$ is the velocity of magnetic bead, and $f_{d}$ is the hydrodynamic resistance coefficient.

$$
v_{p}=\frac{d \tau}{d t}
$$

When the wall effect ${ }^{33}$ is considered, the expression of hydrodynamic resistance coefficient is as follows: 


$$
f_{D}=\left[1-\frac{9}{16}\left(\frac{r}{r+s}\right)+\frac{1}{8}\left(\frac{r}{r+s}\right)^{3}-\frac{45}{256}\left(\frac{r}{r+s}\right)^{4}-\frac{1}{16}\left(\frac{r}{r+s}\right)^{5}\right]^{-1}
$$

Where $s$ is the distance between the magnetic bead and the wall of microchannel. When the magnetic bead is far away from the wall, $f_{D}=1$; When the magnetic bead is near the wall, $f_{D}$ is slightly greater than 1 .

(3) Speed of magnetic bead

The time required for magnetic bead to reach a new equilibrium is extremely small. It can be considered that the motion of magnetic bead in the microchannel is always in a quasi equilibrium state. The motion equation of the magnetic bead can be simplified as:

$$
F_{m}+F_{d}=0
$$

By introducing equations (8) and (9), we get the following results:

$$
v_{p}=\frac{d \tau}{d t}=\frac{1}{6 \pi \eta r f_{D}} F_{m}+v_{f}
$$

The velocity components in each direction can be expressed as:

$$
\left\{\begin{array}{l}
v_{p x}=\frac{d x}{d t}=\frac{1}{6 \pi \eta r f_{D}} F_{m x}+v_{f x} \\
v_{p y}=\frac{d y}{d t}=\frac{1}{6 \pi \eta r f_{D}} F_{m y}+v_{f y} \\
v_{p z}=\frac{d z}{d t}=\frac{1}{6 \pi \eta r f_{D}} F_{m z}+v_{f z}
\end{array}\right.
$$

When the magnetic beads with a certain velocity flows go through the microchannel, the magnetic beads will be shifted and separated from the fluid by magnetic force. Considerring the width to height ratio of the microchannel is large, the separation process can be simplified as a two-dimensional model.

\subsection{Motion analysis of preset magnetic bead capture}

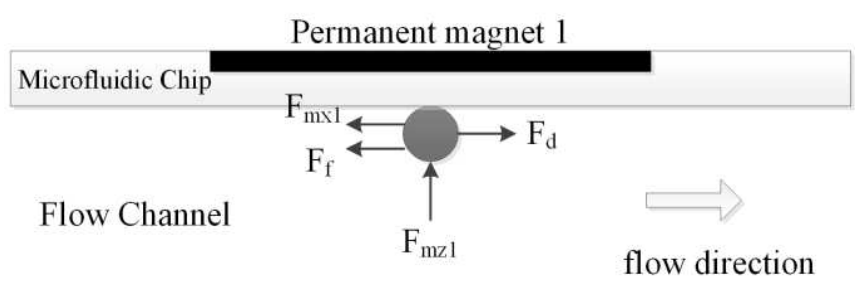

Fig. 9 Preset magnetic bead capture

The capture motion includes magnetic beads captured by permanent magnet 1 (preset permanent magnet) after magnetic beads released and fixed on the upper surface of the channel when the solution washing. The force analysis of magnetic bead capture is shown in Fig. 9, where $F_{f}$ is the friction force between magnetic bead and the channel upper surface. When the magnetic bead is stable in the capture position, $F_{m x l}=0$ and $v_{P}=0$. The condition for the successful capture of magnetic beads under the velocity $v_{f}$ is as follows: 


$$
F_{f}=F_{\mathrm{d}}
$$

In the critical state:

$$
F_{f}=f F_{\mathrm{mz} 1}
$$

Where $f$ is the friction coefficient between the magnetic bead and the flow channel. Introduce equation (8) and equation (15) into equation (14), and get:

$$
F_{m z 1}=\frac{6 \pi \eta r f_{D}}{f} v_{f}
$$

Let $\frac{6 \pi \eta r f_{D}}{f}=[\mathrm{a}]$, When the microfluidic system is determined, $[\mathrm{a}]$ is a certain value, then:

$$
F_{m z 1}=[a] v_{f}
$$

Therefore, only when the z-direction magnetic force produced by permanent magnet 1 is greater than [a] times of the liquid flow rate, the magnetic beads can be effectively captured.

\subsection{Motion analysis of preset magnetic bead mixing}

The mixing motion between magnetic beads and solution can be divided into upward mixing motion and downward mixing motion, which are driven by different permanent magnets. Because of using preset magnetic beads technology, $v_{f z}$ is considered as 0 .

\section{(1) Upward mixing motion}

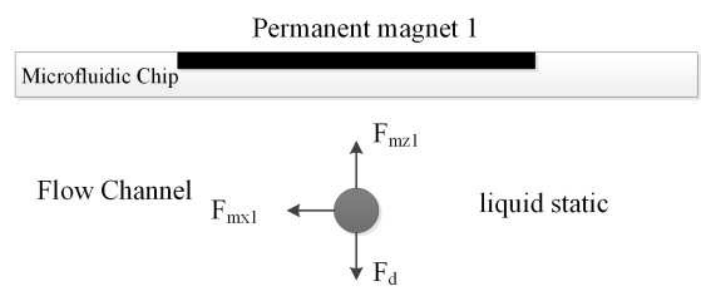

Fig. 10 Upward mixing motion

The upward mixing motion is achieved by permanent magnet 1 . At this time, the permanent magnet 2 falls far enough, so that the influence of its magnetic field on magnetic bead could be ignored. The force analysis of upward mixing motion of magnetic bead is shown in Fig. 10. The achievement of upward mixing motion should satisfy $F_{m z l}>F_{d}$, considering the quasi equilibrium state of magnetic bead motion:

$$
v_{p z 1}=\frac{d z}{d t}=\frac{1}{6 \pi \eta r f_{D}} F_{m z 1}
$$

The microchannel height is $\mathrm{Q}$, and integrate both sides: 


$$
t_{1}=\frac{6 \pi \eta r f_{D} Q}{F_{m z 1}}
$$

Where $t_{l}$ is the time required for upward mixing motion.

\section{(2) Downward mixing motion}

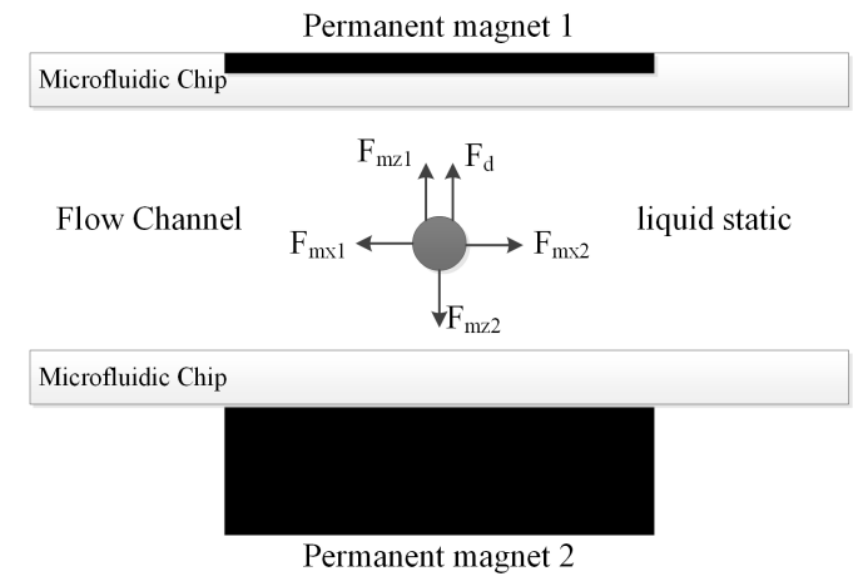

Fig. 11 Downward mixing motion

The downward mixing motion is achieved by permanent magnet 2 . Affected by the magnetic field of permanent magnet 1 , the force analysis of downward mixing motion of magnetic bead is shown in Fig. 11. The achievement of downward mixing motion should satisfy $f_{m z 2}>f_{m z 1}+F_{d}$, considering the quasi equilibrium state of magnetic bead motion:

$$
\left\{\begin{array}{l}
v_{p z 1}=\frac{1}{6 \pi \eta r f_{D}} F_{m z 1} \\
v_{p z 2}=\frac{1}{6 \pi \eta r f_{D}} F_{m z 2}
\end{array}\right.
$$

The downward velocity of magnetic bead is:

$$
v_{p z}=\frac{d z}{d t}=v_{p z 2}-v_{p z 1}=\frac{1}{6 \pi \eta r f_{D}}\left(F_{m z 2}-F_{m z 1}\right)
$$

Integrate both sides:

$$
t_{2}=\frac{6 \pi \eta r f_{D} Q}{F_{m z 2}-F_{m z 1}}
$$

Where $t_{2}$ is the time required for downward mixing motion.

The mixing efficiency of magnetic beads is determined by $t_{1}$ and $t_{2}$. After the magnetic beads confirmed, the mixing efficiency depends on the following factors: (1) The smaller fluid dynamic viscosity, the higher mixing efficiency; (2) The greater magnetic field intensity of preset permanent magnet, the shorter upward mixing motion time, but the downward mixing motion needs to be considered comprehensively; (3) The greater magnetic field intensity difference, the shorter downward mixing motion time; (4) The smaller channel height, the higher mixing efficiency. After determining the type of magnetic beads, the size and quantity of flow channel and 
the type of different reaction solutions, the specification of permanent magnet should be selected reasonably to ensure the magnetic beads motion effect, simplify the device structure and reduce the structure size.

\section{Conclusion}

A magnetic beads preset technology was provided, and the feasibility of its application in microfluidic chip was verified. Based on the analysis of magnetic beads control motion, the motion model of preset magnetic beads was established, including capture motion and mixing motion. The mixing motion was decomposed into upward mixing motion and downward mixing motion, and the relationship between capture condition, mixing time and coupling field was derived, which can provide calculation and design basis for simulation and control of preset magnetic beads. However, in the derivation of mixing time, the influence of movable permanent magnet was completely ignored in the upward mixing motion. And the time of downward mixing motion was calculated based on the movable permanent magnet moving to the closest position to the chip, but the equilibrium position should be located in a certain position during the ascending motion of movable permanent magnet. The actual mixing time should be shorter than the calculation time, that is the more accurate magnetic beads motion control time needing to consider the velocity of movable permanent magnet. In addition, our study ignored the influence of magnetic force in the direction of flow channel, which can be considered in the follow-up study to deeply analyze the distribution trajectory of magnetic beads along the flow channel.

\section{Reference}

1. Hohnholt M C, Geppert M, Dringen R. Treatrent with iron oxide nanop-articles induces ferritin synthesis but not oxidative stress in olig-odendroglial cell. Acta Biomater, 2011,7(11): 3946-3954.

2. Lim C T, Zhang Y. Bead-based microfluidic immunoassays: The next generation. Biosensors \& Bioelectronics, 2007, 22(7): 1197-1204.

3. Safarík I, Safaríková M. Use of magnetic techniques for the isolation of cells. Journal of chromatography. B, Biomedical sciences and applications, 1999, 722:33-53.

4. Safarik I, Safarikova M. Magnetic techniques for the isolation and purification of proteins and peptides. BioMagnetic Research and Technology, 2004, 2: 7.

5. Esmaeili E, Ghiass M A, Vossoughi M, et al. Hybrid magnetic-DNA directed immobilisation approach for efficient protein capture and detection on microfluidic platforms. Scientific Reports, 2017, 7(1): 194.

6. Bao G, Mitragotri S, Tong S. Multifunctional nanoparticles for drug delivery and molecular imaging. Annual Review of Biomedical Engineering, 2013, 15(1): 253-282.

7. Singh A, Sahoo S K. Magnetic nanoparticles: a novel platform for cancer theranostics. Drug Discovery Today, 2014, 19(10): 474-481.

8. Song X, Luo X, Zhang Q, Zhu A, et al. Preparation and characterization of biofunctionalized 
chitosan/Fe3O4 magnetic nanoparticles for application in liver magnetic resonance imaging. Journal of Magnetism and Magnetic Materials, 2015(388): 116-122.

9. Hejazian M, Nguyen N-T. Negative magnetophoresis in diluted ferrofluid flow. Lab on a Chip, 2015, 15(14): 2998-3005.

10. Zhu T, Cheng R, Lee SA, et al. Continuous-flow ferrohydrodynamic sorting of particles and cells in microfluidic devices. Microfluidics and Nanofluidics, 2012, 13(20): 645-654.

11. Xi Z, Huang R, Li Z, et al. Selection of HBsAg-specifc DNA aptamers based on carboxylated magnetic nanoparticles and their application in the rapid and simple detection of hepatitis B virus infection. ACS Applied Materials \& Interfaces, 2015, 7(21): 11215-11223.

12. Alnaimat F, Dagher S , Mathew B , et al. Microfluidics Based Magnetophoresis: A Review. The Chemical Record, 2018, 18(11):1-18.

13. Reenen A V , Jong A M D , Toonder J M J D , et al. Integrated lab-on-chip biosensing systems based on magnetic particle actuation - a comprehensive review. Lab on A Chip, 2014, 14.

14. Myklatun A, Cappetta M, Winklhofer M, et al. Microfluidic sorting of intrinsically magnetic cells under visual control. Scientific Reports, 2017, 7(1): 6942.

15. Cao Q, Liu M, Wang Z, et al. Dynamic motion analysis of magnetic particles in microfluidic systems under an external gradient magnetic field. Microfluidics and Nanofluidics, 2017, 21(2): 24.

16. Pourmehran O, Gorji T B, Gorji-Bandpy M. Magnetic drug targeting through a realistic model of human tracheobronchial airways using computational fluid and particle dynamics. Biomechanics and Modeling in Mechanobiology, 2016, 15(5):1355-1374.

17. Zeng J, Deng Y, Vedantam P, et al. Magnetic separation of particles and cells in ferrofluid flow through a straight microchannel using two offset magnets. Journal of Magnetism and Magnetic Materials, 2013, 346(1): 118-123.

18. Tarn M D, Peyman S A, Robert D, et al. The importance of particle type selection and temperature control for on-chip free-flow magnetophoresis. Journal of Magnetism and Magnetic Materials, 2009, 321(24): 4115-4122.

19. Ghazali F A M , Hasan M N , Rehman T , et al. MEMS actuators for biomedical applications: a review. Journal of Micromechanics and Microengineering, 2020, 30(7):073001-073020.

20. Zhou R, Yang Q, Bai F, et al. Fabrication and integration of microscale permanent magnets for particle separation in microfluidics. Microfluidics and Nanofluidics, 2016, 20(7):1-12.

21. Yang C, Li G. A novel magnet-actuated droplet manipulation platform using a floating ferrofluid film. Scientific Reports, 2017, 7(1):15705.

22. Guckenberger D J, Pezzi H M, Regier M C, et al. Magnetic System for Automated Manipulation of Paramagnetic Particles. Analytical Chemistry, 2016, 88(20):9902-9907.

23. Pamma N . Magnetism and microfluidics. Lab on a Chip, 2006, 6(1):24-38.

24. Antfolk M, Laurell T . Continuous flow microfluidic separation and processing of rare cells and bioparticles found in blood - A review. Analytica Chimica Acta, 2017, 965:9-35.

25. Jayamohan H, Romanov V, Li H, et al. Advances in Microfluidics and Lab-on-a-Chip Technologies. Molecular Diagnostics, 2017:197-217.

26. Schuler F, Trotter M, Geltman M, et al. Digital droplet PCR on disk. Lab on A Chip, 2015, 16(1):208-216.

27. Sun Y, Quyen T L, Hung T Q, et al. A lab-on-a-chip system with integrated sample preparation and loop-mediated isothermal amplification for rapid and quantitative detection of 
Salmonella spp. in food samples. Lab on A Chip, 2015, 15 (8), 1898-1904.

28. Cao W, Bean B, Corey S, et al. Automated Microfluidic Platform for Serial Polymerase Chain Reaction and High-Resolution Melting Analysis. Journal of Laboratory Automation, 2015:402-411.

29. Shi X, Chen C H, Gao W, et al. Parallel RNA extraction using magnetic beads and a droplet array. Lab Chip, 2015, 15(4):1059-1065.

30. Suwa M, Watarai H. Magnetoanalysis of micro/nanoparticles: A review. Analytica Chimica Acta, 2011, 690(2): 137-147.

31. Banerjee U, Bit P, Ganguly R, et al. Aggregation dynamics of particles in a microchannel due to an applied magnetic field. Microfluidics \& Nanofluidics, 2012, 13(4):565-577.

32. Md Ali M A, Ostrikov K, Khalid F A, et al. Active bioparticle manipulation in microfluidic systems. Rsc Advances, 2016, 6(114):113066-113094.

33. Zhou R, Wang C. Microfluidic separation of magnetic particles with soft magnetic microstructures. Microfluid Nanofluid, 2016, 20(3):1-11.

\section{Author Contributions}

Zhao Li and Zhe Du conceived the experiment(s), Xiangyang Zu and Zhigang Hu conducted the experiment, $\mathrm{Zhao} \mathrm{Li}$ and Xiangyang $\mathrm{Zu}$ analysed the results and derived the mathematical model. All authors reviewed the manuscript.

Competing Interests: The authors declare that they have no competing interests. 